\title{
PROTEÇÃO QUÍMICA E BIOLÓGICA DE GRÃOS DE MILHO ÚMIDOS CONTRA FUNGOS DE ARMAZENAGEM E PRODUÇÃO DE AFLATOXINAS
}

\author{
NICÉSIO FILADELFO JANSSEN DE ALMEIDA PINTO ${ }^{1}$; GUILHERME PRADO ${ }^{2}$; MARIZE S. \\ OLIVEIRA $^{2}$
}

\begin{abstract}
${ }^{1}$ Pesquisador, Embrapa Milho e Sorgo, Laboratório de Patologia de Sementes e Grãos, Caixa Postal 151, 35701-970 Sete Lagoas, MG.E-mail:nicesio@cnpms.embrapa.br

${ }^{2}$ Fundação Ezequiel Dias, Núcleo de Micologia e Micotoxinas, Rua Conde Pereira Carneiro, 80, Bairro Gameleira, 30510-010 Belo Horizonte/MG. E-mail: gui@funed.mg.gov.br
\end{abstract}

Revista Brasileira de Milho e Sorgo, v.4, n.2, p.172-179, 2005

RESUMO - Grãos de milho da cultivar HD 9486, com 19,7\% de umidade, foram tratados, imediatamente após a colheita, com soluções de extrato bruto de Eucalyptus citriodora (40\%), essência de Eucalyptus citriodora (20\%), ácido acético (5\%), uréia (5\%), acetato de sódio (5\%), com os fungicidas benomyl (25g i.a.100 $\mathrm{kg}^{-1}$ de grãos), thiabendazole (45g) e fludioxonil (3g); óleo de girassol, óleo mineral e com suspensão de $10^{6}$ UFC. $\mathrm{ml}^{-1}$ de Sacharomyces cerevisiae. Aos grãos da testemunha foi adicionada água, obedecendo à mesma relação utilizada nos demais tratamentos $(0,75 \%$ peso de grãos/volume do produto). A seguir, esses grãos foram armazenados em sistema com aeração contínua, constituído de tambores metálicos de 200 litros de capacidade, com fundo de tela de aço e sobre dutos de aeração, e em sistema sem aeração, constituído de tambores com fundo de metal, sobre estrados de madeira. O delineamento experimental foi o inteiramente casualizado, com 24 tratamentos (parcelas com $80 \mathrm{~kg}$ de grãos), em três repetições. A eficiência dos produtos no controle de Aspergillus flavus e Penicillium spp. foi avaliada quantificando-se a porcentagem de grãos visualmente mofados, aos 17 dias de armazenagem. No sistema sem aeração, os fungicidas benomyl, thiabendazole e fludioxonil apresentaram controles desses fungos a níveis abaixo do limite de tolerância, que é de $3 \%$. A testemunha apresentou $49,6 \%$ de grãos mofados. As médias da umidade e da temperatura intergranular foram de $15,5 \%$ e $35,5^{\circ} \mathrm{C}$, respectivamente. No sistema aerado, todos os tratamentos apresentaram baixo nível de mofamento dos grãos $(1,2 \%$ na testemunha). Provavelmente, nesse período de armazenagem, a temperatura intergranular e a umidade dos grãos foram desfavoráveis ao desenvolvimento desses fungos. Nessa época, foram determinados os níveis ( $\mu \mathrm{g} . \mathrm{kg}^{-1}$ ou ppb) de aflatoxinas, sendo utilizado o métodos ELISA e cromatografia em camada delgada. Na condição aerada, os grãos de milho apresentaram os menores níveis de aflatoxinas e foram significativamente diferentes $(\mathrm{P}<0,05)$, quando comparados aos valores encontrados na condição não aerada. Na condição aerada, a maior eficiência na proteção dos grãos de milho em relação à biossíntese de aflatoxinas $\left(2,1 \mu \mathrm{g} \cdot \mathrm{kg}^{-1}\right.$ de $\left.\mathrm{B}_{1}+\mathrm{B}_{2}+\mathrm{G}_{1}+\mathrm{G}_{2}\right)$ foi verificada no tratamento com thiabendazole. Do exposto, conclui-se que a aeração contínua e a proteção dos grãos com os fungicidas benomyl, thiabendazole e fludioxonil são eficientes contra o mofamento causado por Aspergillus flavus e Penicillium spp. e na redução da biossíntese de aflatoxinas.

Palavras-chave: Zea mays, fungos toxigênicos, micotoxinas, patologia de grãos. 


\title{
CHEMICAL AND BIOLOGICAL PROTECTION OF HUMID CORN GRAINS AGAINST STORAGE FUNGI AND AFLATOXINS PRODUCTION
}

\begin{abstract}
Grains of corn cultivars HD 9486 with 19,7\% moisture were treated, immediately after harvest, with solutions of Eucalyptus citriodora extract (40\%), Eucalyptus citriodora essence (20\%), acetic acid (5\%), uréia (5\%), sodium acetate (5\%); benomyl (25g a.i. $100 \mathrm{~kg}^{-1}$ of grains), thiabendazole (45g) and fludioxonil (3g), sunflower oil, mineral oil and suspension of $10^{6} \mathrm{CFU} \cdot \mathrm{ml}^{-1}$ of Sacharomyces cerevisiae. To the check grains , water was added obeying the same relationship used in other treatments $(0.75 \%$ grain weight /product volume). Those grains were stored in a continuous aeration system, constituted of 200 liter metallic drums with steel screen bottoms on ducts of aeration; and in a non-aeration system, constituted of metal bottom drums on wood bases. The experimental design was entirely randomized with 24 treatments (plots with $80 \mathrm{~kg}$ of grains) in 3 replications. The efficiency in the control of Aspergillus flavus and Penicillium spp. was evaluated being quantified the percentage of grains visually moldy after 17 days of storage. In the non-aeration system, the fungicides benomyl, thiabendazole and fludioxonil controlled those fungi at levels below the tolerance limit, that is, $3 \%$, while the check presented $49.6 \%$ of moldy grains. The moisture averages and the temperature between grains were $15.5 \%$ and $35.5{ }^{\circ} \mathrm{C}$, respectively. In the aerated system all treatments presented low level of moldy grains ( $1.2 \%$ in the check), suggesting that in that storage period the temperature and grains moisture were unfavorable to the development of those fungi. At the time, aflatoxins levels $\left(\mu \mathrm{g} . \mathrm{kg}^{-1}\right)$ were evaluated, being used the ELISA and the thin layer chromatographic methods. In the aerated condition, the corn grains presented the smallest aflatoxins levels and were significantly different $(\mathrm{P}<0,05)$ when compared with values found in non-aerated condition. In aerated condition, the largest efficiency in the protection of the corn grains in relation to the aflatoxins biosynthesis $\left(2.1 \mu \mathrm{g} \cdot \mathrm{kg}^{-1}\right.$ of $\mathrm{B} 1+\mathrm{B} 2+\mathrm{G} 1+\mathrm{G} 2$ ) was verified in the treatment with thiabendazole. It is concluded that the continuous aeration and the grains protection with benomyl, thiabendazole and fludioxonil are efficient against the grain moldy caused by Aspergillus flavus and Penicillium spp. and in the reduction of the aflatoxins biosynthesis.
\end{abstract}

Key words: Zea mays, toxigenics fungi, mycotoxins, grain pathology.

Vários são os fatores que influenciam o desenvolvimento de fungos e a produção de micotoxinas em alimentos, sendo a temperatura e a umidade (atividade de água e teor de umidade) dois parâmetros fundamentais nesse processo de biossíntese (Diener \& Davis, 1967).

$\mathrm{Na}$ espera pela secagem de grãos, a prevenção contra o mofamento dependerá de agentes inibidores do desenvolvimento fúngico. Herting \& Drury (1974) verificaram a atividade antifúngica dos ácidos fórmico, acético, propiônico, butílico e isobutílico em grãos de milho. Vandegraft et al. (1975) trataram grãos de milho com $2 \%$ de amônia ou $1 \%$ de ácido propiônico e observaram uma redução significativa no desenvolvimento de mofo. Os fungicidas benomyl e thiabendazole foram utilizados por White et al. (1993) para proteger grãos de milho úmidos durante a secagem com ar ambiente e na armazenagem e reduziram a incidência de Penicillium spp. e Aspergillus spp. A eficácia de iprodione no controle de fungos em grãos de 
milho úmido foi determinada por Toman Jr. \& White (1994).

Pinto $(2001,2004)$ relata que óleo mine$\mathrm{ral}(1 \% \mathrm{p} / \mathrm{v})$ protegeu grãos de sorgo úmidos contra o mofamento causado pelos fungos Aspergillus spp. e Penicillium spp. e que, na espera pela secagem de grãos de milho, os tratamentos desses grãos com ácido acético ou uréia foram eficientes no controle do mofamento causado por Aspergillus flavus. Adicionalmente, segundo Jones \& Prusky (2002), a expressão de um peptídio antifúngico em Saccharomyces cerevisiae resultou na inibição da podridão do fruto de tomate causada por Colletotrichum coccodes. Por outro lado, Franco e Bettiol (2000), visando selecionar produtos alternativos para o controle de Penicillium digitatum em pós-colheita de citros, avaliaram, entre outros produtos, os óleos de soja e de eucalipto e Saccharomyces cerevisiae, em comparação com os fungicidas thiabendazole, prochloraz e imazalil.

No Brasil, o fungicida Tecto 600 (thiabendazole) é o único registrado no MAPA para o tratamento de grãos de milho imediatamente após a colheita, visando, na armazenagem, o controle das podridões causadas por Aspergillus flavus, Penicillium digitatum e Fusarium moniliforme (Tecto, 1999).

Com relação às micotoxinas, as aflatoxinas são metabólitos secundários produzidos por fungos do gênero Aspergillus, que contaminam as culturas no campo, os grãos durante o armazenamento e também os produtos alimentícios destinados ao consumo humano (Who, 1979). Devido aos efeitos carcinogênicos, mutagênicos e teratogênicos, as aflatoxinas representam um risco à saúde pública. Condições ambientais favoráveis, associadas à suscetibilidade dos grãos de milho, maximizam a produção das aflatoxinas, implicando a necessidade de um monitoramento constante e desenvolvimento de técnicas de cultivo, colheita, pós-colheita, armazenamento e transporte.

Diener \& Davis (1967) e Schindler et al. (1967) verificaram que a temperatura mínima de produção de aflatoxina é de $12^{\circ} \mathrm{C}$ e a máxima de $42{ }^{\circ} \mathrm{C}$, sendo a ótima entre 24 e $30^{\circ} \mathrm{C}$. Com relação à umidade relativa, o limite máximo é de $85 \%$. O substrato deve apresentar atividade de água de no mínimo 0,85 para que o fungo possa produzir a toxina. Em milho, foi verificado que a atividade de água que mais favorece a produção de aflatoxina por Aspergillus parasiticus é de 0,90 (Montani \& Vaamond, 1988).

O milho é um bom substrato para o desenvolvimento de fungos e produção de aflatoxinas (Sabino et al., 1986; Sabino et al., 1989; Shotwell \& Hesseltine, 1983; Stoloff et al., 1976).

Prado et al. (1995) relatam uma redução dos níveis de aflatoxinas em amostras de grãos de milho da cultivar BR 201, com diferentes níveis de umidade $(25,8 ; 22,9 ; 18,8 ; 17,7 ; 16,1 \mathrm{e}$ $11,7 \%$ ), tratadas com fungicida (iprodiona) e armazenadas em atmosfera aerada, com temperatura intergranular de $18-20^{\circ} \mathrm{C}$. Quando o acondicionamento foi realizado em atmosfera sem aeração, o decréscimo dos teores de aflatoxinas não foi significativo, indicando que a temperatura $\left(35-40^{\circ} \mathrm{C}\right)$ e a umidade são fatores importantes na biossíntese de aflatoxinas.

O objetivo deste trabalho foi avaliar a eficiência da proteção química e biológica de grãos de milho úmidos contra o mofamento incitado por Aspergillus flavus e Penicillium spp., bem como minimizar ou eliminar a biossíntese de aflatoxinas em sistemas de armazenagem com aeração e sem aeração.

\section{Material e Métodos}

Grãos de milho da cultivar HD 9486 foram colhidos com $19,7 \%$ de umidade e 
imediatamente tratados, em tratador mecânico de grãos/sementes, com os seguintes produtos: P1- extrato bruto de Eucalyptus citriodora solução a 40\%; P2- essência de Eucalyptus citriodora solução a 20\%; P3- ácido acético solução a 5\%; P4- uréia solução a 5\%; P5- acetato de sódio solução a 5\%; P6- benomyl (25g i.a.100 $\mathrm{kg}^{-1}$ de grãos); P7- thiabendazole (45g); P8- fludioxonil (3g); P9óleo de girassol a $0,75 \%$ peso de grãos/volume do produto; P10- óleo mineral $0,75 \%$ peso de grãos/volume do produto; P11- com suspensão de $10^{6}$ CFU.ml ${ }^{-1}$ de Sacharomyces cerevisiae. Aos grãos da testemunha (P12) foi adicionada água, obedecendo à mesma relação utilizada nos demais tratamentos $(0,75 \%$ peso de grãos/volume do produto). A seguir, os grãos (80 kg por parcela) foram armazenados em tambores metálicos de 200 litros de capacidade, com fundo de tela de aço e sobre dutos de aeração, e em sistema sem aeração, em tambores com fundo de metal. Os tambores tinham dimensões de $0,82 \mathrm{~m}$ de altura e $0,60 \mathrm{~m}$ de diâmetro. O sistema com aeração (Figura 1)

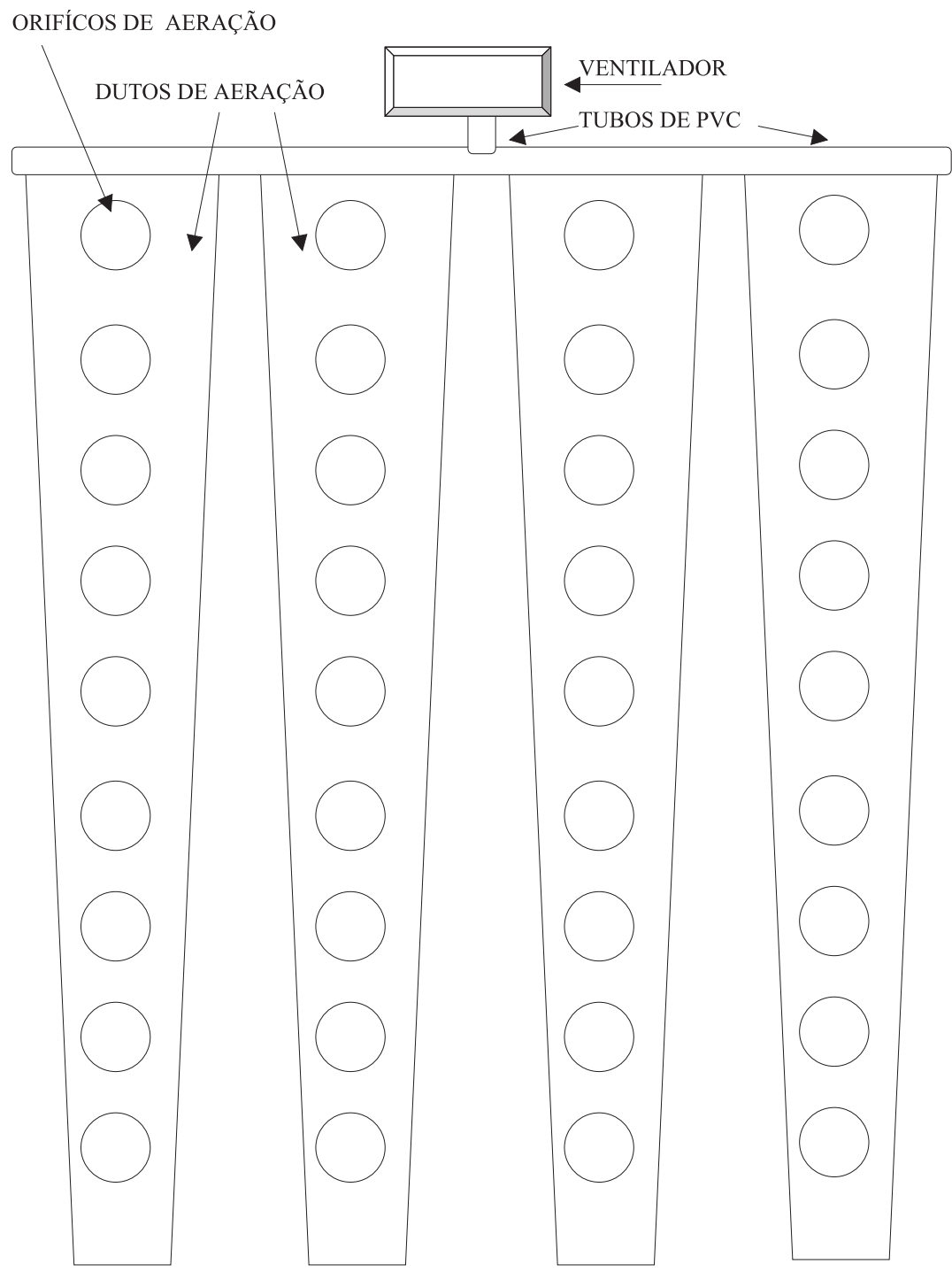

FIGURA 1. Sistema com aeração contínua, com ar ambiente 
foi dotado de quatro dutos com formato trapezoidal $(6,0 \times 1,1 \times 0,7 \mathrm{~m})$, possuindo cada duto nove orifícios de aeração ( $0,54 \mathrm{~m}$ de diâmetro), sobre os quais foram colocados os tambores com a massa de grãos de milho. A aeração contínua com ar ambiente (20$30{ }^{\circ} \mathrm{C}$ ) foi promovida por um ventilador de 2750 rpm, motor de $0,5 \mathrm{HP}$, vazão de ar de $0,42 \mathrm{~m}^{3} / \mathrm{seg}$. $\mathrm{O}$ delineamento foi o inteiramente casualizado, com 24 tratamentos em três repetições.

O controle de Aspergillus flavus e Penicillium spp. foi avaliado quantificando-se a porcentagem de grãos visualmente mofados aos $3,5,7,10,12,14$ e 17 dias de armazenagem. A umidade dos grãos foi determinada nesses períodos $\left(105+2{ }^{\circ} \mathrm{C} / 24 \mathrm{~h}\right)$, sendo o monitoramento da temperatura intergranular realizado diariamente.

Visando determinar, em pós-colheita, a produção de aflatoxinas em grãos de milho por Aspergillus flavus, aos 17 dias de armazenagem foram coletadas amostras de $2,0 \mathrm{~kg}$ de grãos nas 72 parcelas experimentais e enviadas ao Laboratório de Micotoxinas da Fundação Ezequiel Dias - FUNED, em Belo Horizonte, MG.
A determinação de aflatoxinas foi feita utilizando o método de ELISA. As amostras em grãos de cada parcela foram moídas, homogeneizadas e passadas em tamis 20 mesh. Foram utilizados kits da Neogen Corporation (USA) para determinação direta e competitiva de aflatoxina total, de acordo com o procedimento analítico descrito pelo fabricante e dentro do prazo de validade especificado. A confirmação dos resultados positivos foi feita pela técnica de cromatografia em camada delgada, utilizando-se a metodologia descrita por Soares \& Rodrigues-Amaya (1989), com quantificação densitométrica.

Para verificar a eficiência dos tratamentos sobre a biossíntese de aflatoxinas, foi realizada a análise de variância dos dados e as médias, comparadas pelo teste $t$ de Student $(\mathrm{p}<0,05)$.

\section{Resultados e Discussão}

No sistema sem aeração (Tabela 1), até os 17 dias de armazenagem, os produtos benomyl, fludioxonil e thiabendazole apresentaram controle de fungos a níveis abaixo do

TABELA 1. Monitoramento da umidade, da temperatura e do mofamento de grãos úmidos de milho da cultivar HD 9486, colhidos com 19,7\% de umidade e imediatamente tratados com substâncias químicas, biológicas e extratos vegetais e armazenados por 17 dias em sistemas com e sem aeração. Embrapa Milho e Sorgo, Sete Lagoas, MG, 1999.

\begin{tabular}{|c|c|c|c|c|c|c|}
\hline \multirow[b]{2}{*}{ Produto * } & \multicolumn{2}{|c|}{ Umidade (\%) } & \multicolumn{2}{|c|}{ Temperatura $\left({ }^{\circ} \mathrm{C}\right)$} & \multicolumn{2}{|c|}{ Mofamento (\%) } \\
\hline & SCA $^{* *}$ & SSA & SCA & SSA & SCA & SSA \\
\hline $\mathrm{P} 1$ & 14,4 & 15,6 & 23,7 & 36,3 & 0,8 & 35,2 \\
\hline $\mathrm{P} 2$ & 14,1 & 14,8 & 22,7 & 37,3 & 0,3 & 24,1 \\
\hline P3 & 15,5 & 15,9 & 23,0 & 38,7 & 2,2 & 27,6 \\
\hline P4 & 13,9 & 16,3 & 22,0 & 38,0 & 0,9 & 21,9 \\
\hline P5 & 13,1 & 16,9 & 23,3 & 35,0 & 0,9 & 58,8 \\
\hline P6 & 13,7 & 17,3 & 22,7 & 28,0 & 0,0 & 1,7 \\
\hline P7 & 13,8 & 18,5 & 22,7 & 32,0 & 0,0 & 2,8 \\
\hline P8 & 13,5 & 16,6 & 23,3 & 34,0 & 0,6 & 2,1 \\
\hline P9 & 13,9 & 15,8 & 23,3 & 37,7 & 1,0 & 21,6 \\
\hline P10 & 13,7 & 16,8 & 23,0 & 37,3 & 0,7 & 21,7 \\
\hline P11 & 14,2 & 15,5 & 22,3 & 36,3 & 0,9 & 30,6 \\
\hline $\mathrm{P} 12$ & 13,5 & 16,4 & 22,7 & 35,7 & 1,2 & 49,6 \\
\hline Média & 13,9 & 16,3 & 22,9 & 35,5 & & \\
\hline
\end{tabular}

* P1 - Extrato bruto de Eucalyptus citriodora, P2 - Essência de Eucalyptus citriodora, P3 - Ácido acético, P4 - Uréia, P5 - Acetato de sódio, P6 - Benomyl, P7 - Thiabendazole, P8 - Fludioxonil, P9 - Óleo de girassol, P10 - Óleo mineral, P11 - Sacharomyces cerevisiae, e P12 - Testemunha.

** SCA - Sistema com aeração e SSA - Sistema sem aeração. 
limite de tolerância, que é de $3 \%$. A testemunha apresentou $49,6 \%$ de mofamento. Esses resultados estão de acordo com os relatados por White et al. (1993), White \& Toman Jr. (1994) e Toman Jr. \& White (1994). Ainda nesse sistema, as médias da umidade e da temperatura intergranular foram, respectivamente, de 16,3\% e $35,5{ }^{\circ} \mathrm{C}$. Por outro lado, no sistema com aeração, essas médias foram, respectivamente, de $13,9 \%$ e $22,9^{\circ} \mathrm{C}$, impedindo o desenvolvimento dos fungos de armazenagem.

No sistema com aeração (Tabela 1), a umidade variou de 13,1 a $15,5 \%$ e a temperatura variou de 22,0 a $23,7^{\circ} \mathrm{C}$. No sistema sem aeração, houve uma variação de 14,8 a 18,5\% na umidade e de 28,0 a $38,7{ }^{\circ} \mathrm{C}$ na temperatura. Assim, no sistema com aeração, todos os tratamentos, inclusive a testemunha $(1,2 \%)$, apresentaram baixo nível de mofamento dos grãos, sugerindo que a temperatura intergranular e a umidade dos grãos foram desfavoráveis ao desenvolvimento de Aspergillus flavus e de Penicillium spp.

Com relação aos resultados obtidos nas análises de micotoxinas, na Tabela 2 são apresentados os níveis de aflatoxinas encontrados nas amostras de milho tratadas com os diversos

TABELA 2. Níveis de aflatoxina B1 + B2 + G1 + G2 ( $\mu \mathrm{g} \cdot \mathrm{kg}^{-1}$ ou ppb) em grãos de milho tratados com produtos químicos e biológicos e armazenadas por 17 dias em sistemas com aeração e sem aeração. Embrapa Milho e Sorgo, Sete Lagoas, MG, 1999.

\begin{tabular}{cll}
\hline Produto * & Sistema com aeração & Sistema sem aeração \\
\hline P1 & $104,7 \mathrm{deA}^{* *}$ & $5926,7 \mathrm{cdB}$ \\
P2 & $36,1 \mathrm{bcA}$ & $3943,3 \mathrm{bcdB}$ \\
P3 & $31,1 \mathrm{bcdA}$ & $4280,0 \mathrm{bcdB}$ \\
P4 & $37,3 \mathrm{cdeA}$ & $4083,3 \mathrm{bcdB}$ \\
P5 & $253,2 \mathrm{eA}$ & $6626,7 \mathrm{~dB}$ \\
P6 & $28,0 \mathrm{abA}$ & $632,5 \mathrm{aB}$ \\
P7 & $2,1 \mathrm{aA}$ & $1283,5 \mathrm{abcB}$ \\
P8 & $49,0 \mathrm{cdeA}$ & $989,3 \mathrm{abB}$ \\
P9 & $174,8 \mathrm{eA}$ & $2640,0 \mathrm{abcdB}$ \\
P10 & $38,4 \mathrm{cdeA}$ & $2973,3 \mathrm{bcdB}$ \\
P11 & $84,5 \mathrm{cdeA}$ & $5080,0 \mathrm{cdB}$ \\
\hline P12 & $36,5 \mathrm{bcdA}$ & $5133,3 \mathrm{cdB}$ \\
\hline
\end{tabular}

* Relacionado na Tabela 1.

** Médias seguidas da mesma letra minúscula, na mesma coluna e maiúscula, na mesma linha, não diferem significativamente entre si, pelo teste t, de Student $(\mathrm{P}<0,05)$. 
produtos químicos e biológicos e armazenadas nos sistemas com aeração e sem aeração. Observa-se que o sistema com aeração foi significativamente diferente $(\mathrm{p}<0,05)$ do sistema sem aeração, em todos os tratamentos aplicados. Constata-se que, nos grãos sem tratamento (P12testemunha), quando armazenados no sistema com aeração, os níveis de aflatoxinas foram superiores aos encontrados nas amostras tratadas com o fungicida thiabendazole (P7), indicando o efeito protetor do tratamento realizado. Esse resultado confirma os relatos de Prado et al. (1995), quando da utilização do fungicida iprodiona em grãos úmidos de milho. Os níveis de aflatoxinas encontrados $\left(2,1 \mu \mathrm{g} . \mathrm{kg}^{-1}\right.$ ou ppb de B1 + B2 + $\mathrm{G} 1+\mathrm{G} 2$ ) nos grãos tratados com thiabendazole (P7) e armazenados no sistema com aeração foram inferiores aos exigidos pela Resolução RDC n. 274/2002 da Agência Nacional de Vigilância Sanitária - ANVISA, do Ministério da Saúde (20 $\mu \mathrm{g} \cdot \mathrm{kg}^{-1}$ ou ppb de B1 + B2 + G1 + G2).

No sistema sem aeração, níveis elevados de aflatoxinas foram observados em todos os tratamentos (variando de 632,5 a 6626,7 $\mu \mathrm{g} . \mathrm{kg}^{-1}$ ), sendo as menores contaminações obtidas nos grãos tratados com os fungicidas benomyl $(632,5$ $\left.\mu \mathrm{g} . \mathrm{kg}^{-1}\right)$, fludioxonil $\left(989,3 \mu \mathrm{g} \cdot \mathrm{kg}^{-1}\right)$ e thiabendazole (1283,5 $\left.\mu \mathrm{g} \cdot \mathrm{kg}^{-1}\right)$. Resultados semelhantes foram obtidos por Prado et al. (1995), em grãos de milho colhidos com $18,8 \%$ de umidade, tratados com iprodiona e armazenados em atmosfera não aerada.

\section{Conclusões}

A aeração contínua e a proteção dos grãos com os fungicidas benomyl, thiabendazole e fludioxonil são procedimentos eficientes contra o mofamento incitado pelos fungos Aspergillus flavus e Penicillium spp., bem como na redução da biossíntese de aflatoxinas.

\section{Agradecimentos}

A Osni Alves da Silva, Ademar Verneque e José Moreira Campos (Assistentes de Pesquisa), Gilberto Ribeiro Rodrigues e Almir Roberto da Silva (Operários Rurais) e à Fundação Ezequiel Dias - FUNED, Núcleo de Micologia e Micotoxinas, a colaboração na condução deste trabalho.

\section{Literatura Citada}

DIENER, U. L.; DAVIS, N. D. Limiting temperature and relative humidity for growth and production of aflatoxin and free fatty acids by Aspergillus flavus in sterile peanuts. Journal of the American Oil Chemists Society, Champaign, v. 44, p. 259-263, 1967.

FRANCO, D. A. S.; BETTIOL, W. Controle de Penicillium digitatum em pós-colheita de citros com produtos alternativos. Fitopatologia Brasileira, Brasília, v. 25, n. 4, p. 602-606, 2000.

HERTING, D. C.; DRURY, E. E. Antifungal activity of volatile fatty acids on grains. Cereal Chenistry, St. Paul, v. 51, n. 1, p. 74-83, 1974.

JONES, R. W.; PRUSKY, D. Expression of an antifungal peptide in Saccharomyces: A new approach for biological control of the postharvest disease caused by Colletotrichum coccodes. Phytopathology, St. Paul, v. 92, n. 1, p. 33-37, 2002.

MONTANI, M. L.; VAAMOND, G. Water activity influence on aflatoxin accumulation in corn. International Journal of Food Microbiology, Amsterdam, v. 6, p. 349-355, 1988.

PINTO, N. F. J. A. Tratamento químico de grãos de sorgo úmidos visando o controle de fungos de armazenamento. Revista Brasileira de 
Armazenamento, Viçosa, v. 26, n. 2, p. 55-59, 2001.

PINTO, N. F. J. A. Preservação da qualidade sanitária de grãos úmidos de milho através do controle químico da atividade fúngica. Revista Brasileira de Armazenamento, Viçosa, v. 29, n. 2, p. 159164, 2004.

PRADO, G.; PINTO, N. F. J. A.; OLIVEIRA, M. S. Incidência de aflatoxinas em milho (Zea mays L.) com diferentes níveis de umidade, após tratamento com fungicida, armazenado em atmosfera com e sem aeração. Revista do Instituto Adolfo Lutz, São Paulo, v. 55, n. 2, p. 79-84, 1995.

SABINO, M.; PRADO, G.; COLEN, G. Ocorrência de aflatoxinas, ochratoxina $A$ e zearalenona em milho de Minas Gerais. Parte I. Revista do Instituto Adolfo Lutz, São Paulo, v. 46, p. 65-71, 1986.

SABINO, M.; PRADO, G.; INOMATA, F. I.; PEDROSO, M. O.; GARCIA, R. V. Natural occurence of aflatoxins and zearalenone in maize in Brazil. Part II. Food Additives and Contaminants, Hants, v. 6, p. 327-331, 1989.

SCHINDLER, A. F.; PALMER, J. G.; EISENBERG, W. V. Aflatoxin production by Aspergillus flavus as related to various temperatures. Applied Microbiology, Washington, v. 15, p. 1006-1009, 1967.

SHOTWELL, O. L.; HESSELTINE, C. W. Fiveyears study of mycotoxins in Virginia wheat and dent corn. Journal of the Association Official Analytical Chemists, Washington, v. 6, p. 14661469, 1983.

SOARES, L. M. V.; RODRIGUES-AMAYA, D. B. Survey of aflatoxins, ochratoxin A, zearalenone and sterigmatocystin in brazilian foods by using multi-toxin thin-layer chromatographic methods. Journal of the Association Official Analytical Chemists, Washington, v. 73, p. 2226, 1989.

STOLOFF, L.; HENRY, S.; FRANCIS, O. J. Survey for aflatoxins and zearalenone in 1973 crop corn stored on farms and in country elevatores. Journal of the Association Official Analytical Chemists, Washington, v. 59, p. 119121, 1976.

TECTO 600 - Novartis Biociências S. A. In: ANDREI, E. Compêndio de defensivos agrícolas: guia prático de produtos fitossanitários para uso agrícola. 6. ed. rev. atual. São Paulo: Organização Andrei, 1999. p. 551-553.

TOMAN JR., J.; WHITE, D. G. Efficacy of iprodione for control of storege fungi in corn. Plant Disease, St. Paul, v. 78, n. 1, p. 27-33, 1994.

VANDEGRAFT, E. E.; HESSELTINE, C. W.; SHOTWELL, O. L. Grain preservatives : Effects on aflatoxin and ochratoxin production. Cereal Chemistry, St. Paul, v. 52, n. 1, p. 79-84, 1975.

WHITE, D. G.; TOMAN JR., J. Effects of postharvest oil and fungicide application on storage fungi in corn following high-temperature grain drying. Plant Disese, St. Paul, v. 78, n. 1, p. 38-43, 1994.

WHITE, D. G.; TOMAN JR., J.; BURNETTE, D. C. The effect of postharvest fungicide application on storage fungi of corn during ambient air drying and storage. Plant Disease, St. Paul, v. 77, n. 6, p. 562-568, 1993.

WORLD HEALTH ORGANIZATION (Geneve). Mycotoxins. Geneve, 1979. 127 p. (WHOEnvironmental Health Criteria, 11). 